\title{
G-triplex structure and formation propensity
}

\author{
Linda Cerofolini ${ }^{1,2}$, Jussara Amato ${ }^{3}$, Andrea Giachetti ${ }^{1}$, Vittorio Limongelli ${ }^{3}$, \\ Ettore Novellino ${ }^{3}$, Michele Parrinello ${ }^{4,5}$, Marco Fragai ${ }^{1,6}$, Antonio Randazzo ${ }^{3, *}$ and \\ Claudio Luchinat ${ }^{1,6,{ }^{*}}$
}

\author{
${ }^{1}$ Magnetic Resonance Center (CERM), University of Florence, Sesto Fiorentino, Florence 50019, Italy, ${ }^{2}$ Giotto \\ Biotech, Via Madonna del Piano 6, Sesto Fiorentino, Florence 50019, Italy, ${ }^{3}$ Department of Pharmacy, University of \\ Naples 'Federico II', Naples I-80131, Italy, ${ }^{4}$ Department of Chemistry and Applied Biosciences, ETH Zurich, Zurich \\ $\mathrm{CH}-8006$, Swizerland, ${ }^{5}$ Facoltà di Informatica, Istituto di Scienze Computazionali (ICS), Università della Svizzera \\ Italiana, Lugano $\mathrm{CH}-6900$, Switzerland and ${ }^{6}$ Department of Chemistry, University of Florence, Sesto Fiorentino, \\ Florence 50019, Italy
}

Received July 25, 2014; Revised October 15, 2014; Accepted October 17, 2014

\begin{abstract}
The occurrence of a G-triplex folding intermediate of thrombin binding aptamer (TBA) has been recently predicted by metadynamics calculations, and experimentally supported by Nuclear Magnetic Resonance (NMR), Circular Dichroism (CD) and Differential Scanning Calorimetry (DSC) data collected on a $3^{\prime}$ end TBA-truncated 11-mer oligonucleotide (11-mer$3^{\prime}$-t-TBA). Here we present the solution structure of 11-mer-3'-t-TBA in the presence of potassium ions. This structure is the first experimental example of a G-triplex folding, where a network of Hoogsteen-like hydrogen bonds stabilizes six guanines to form two G:G:G triad planes. The G-triplex folding of 11-mer-3'-t-TBA is stabilized by the potassium ion and destabilized by increasing the temperature. The superimposition of the experimental structure with that predicted by metadynamics shows a great similarity, with only significant differences involving two loops. These new structural data show that 11-mer-3'-t-TBA assumes a G-triplex DNA conformation as its stable form, reinforcing the idea that G-triplex folding intermediates may occur in vivo in human guanine-rich sequences. NMR and CD screening of eight different constructs obtained by removing from one to four bases at either the $3^{\prime}$ and the $5^{\prime}$ ends show that only the 11-mer-3'-t-TBA yields a relatively stable G-triplex.
\end{abstract}

\section{INTRODUCTION}

The structural polymorphism of DNA interplays with nuclear proteins to regulate many cellular activities $(1,2)$. Guanine-rich sequences at the end of human telomeres have been reported to fold into a variety of topologies, including single or multi-stranded G-quadruplex structures (3-6), depending on lengths, number of $\mathrm{G}$ tracts, cation identity and loop sequences. Also the bases sequence at $5^{\prime}$ and/or $3^{\prime}$ end influence the folding topology by forming base-stacking interactions with the core of the quadruplex structure. Gquadruplex structures have been characterized in vitro since the 1990s but only recently have been observed in human cells (7). The role of G-quadruplexes in cell replication makes G-quadruplex structures attractive drug-target candidates for cancer therapy $(8,9)$. The growing importance of G-quadruplex structures has prompted the analysis of their folding mechanism and the search of new polymorphisms involving the guanine-rich sequences. G-quadruplex structures can be formed by a single oligonucleotide or by properly assembled two to four different oligonucleotides stabilized by Hoogsteen-like hydrogen bonds and base stacking (10-12). Several spectroscopic studies show that some guanine-rich sequences fold into compact intramolecular quadruplex structures by following a multistep pathway that proceeds through stable triplex intermediates (13-15). However, none of such triplex structures has been solved by nuclear magnetic resonance (NMR) or X-ray crystallography so far, because they are elusive and difficult to isolate.

Thrombin binding aptamer (TBA) is a DNA 15-mer oligonucleotide, namely, 5'-GGTTGGTGTGGTTGG-3', that folds in a monomeric G-quadruplex structure and binds $\alpha$-thrombin (10), a trypsin-like serine protease involved in hemostasis. In the chair-like structure of the Gquadruplex, two G-tetrads, which are connected by two TT loops and by a single TGT loop, coordinate a monovalent

\footnotetext{
${ }^{*}$ To whom correspondence should be addressed. Claudio Luchinat. Tel: +39055 4574296; Fax: +39055 4574923; Email: claudioluchinat@cerm.unifi.it Correspondence may also be addressed to Antonio Randazzo. Tel/Fax: +39081678514; Email: antonio.randazzo@unina.it

(C) The Author(s) 2014. Published by Oxford University Press on behalf of Nucleic Acids Research.

This is an Open Access article distributed under the terms of the Creative Commons Attribution License (http://creativecommons.org/licenses/by-nc/4.0/), which permits non-commercial re-use, distribution, and reproduction in any medium, provided the original work is properly cited. For commercial re-use, please contact journals.permissions@oup.com
} 


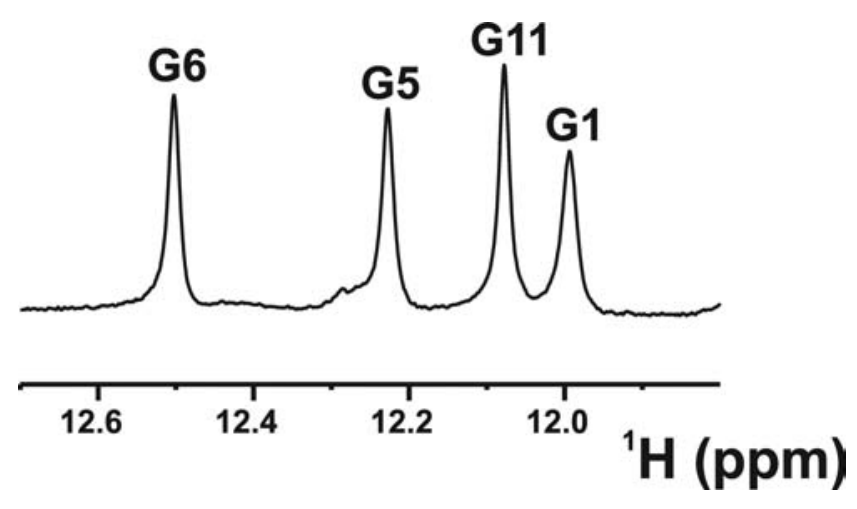

Figure 1. Imino protons region of the ${ }^{1} \mathrm{H}$ NMR spectrum, acquired at 900 $\mathrm{MHz}$ (proton Larmor frequency) and $274 \mathrm{~K}$.

metal ion $(10,16)$. Metadynamics simulations of the TBA unfolding pathway suggested the presence of three main energy minima (17). The deepest one corresponds to the wellcharacterized G-quadruplex structure of TBA (18), which evolves toward a second minimum where TBA shows a partial opening of the $3^{\prime}$ end with residue G15 oriented into the solvent (17). Following the proposed unfolding pathway, a third minimum is encountered, where the $3^{\prime}$ end opens completely with G14 and G15 leaving the G-tetrad planes, which rearrange to form two G:G:G planes (17). To prove the intrinsic stability of the G-triplex, the TBA oligonucleotide was shortened by four residues at the $3^{\prime}$ end (truncated TBA, 11-mer-3'-t-TBA hereafter) and its structural features were investigated in solution. The analysis of the NMR spectra showed the presence of a predominant folded structure in equilibrium with the unfolded strand, and with the structured form highly favored at low temperature. In particular, the presence of four well-defined exchangeable proton signals in the $11.0-12.5 \mathrm{ppm}$ region, which are typical of DNA structures with Hoogsteen-like hydrogen bonds (4), were observed in the spectra (Figure 1). Moreover, a network of NOE (Nuclear Overhauser Effect) interactions between imino protons was consistent with the presence of two G-triads, thus supporting the predictions of molecular dynamics simulations. An important point to address is whether other TBA truncations are capable of yielding a stable G-triplex structure. To this end, a total of eight different constructs lacking from one to four bases at either the $3^{\prime}$ or the $5^{\prime}$ end have been screened by NMR and circular dichroism (CD) spectroscopies. Only the 11-mer-3'-t-TBA was found to be a single G-triplex species with a relatively high stability.

An experimental structure of 11-mer-3'-t-TBA at atomic detail is needed at this point to answer further questions raised by metadynamics prediction. Among them, it is important to clarify how the absence of the four bases at $3^{\prime}$ end of the guanine-rich stretch of TBA, which prevents the formation of a normal G-quadruplex structure, affects the G-triplex structure predicted starting from the whole TBA (Figure 2). The experimentally validated Hoogsteen-like hydrogen bonds among G-triads in the G-triplex folding predicted by metadynamics calculations are shown in Figure 3 (17).
A)

ก $90^{\circ}$

B)

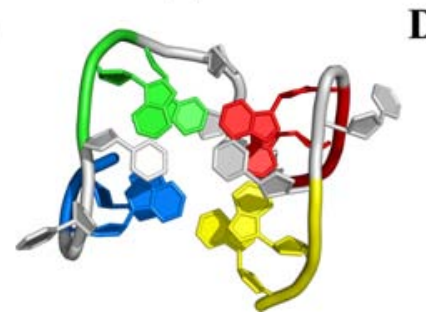

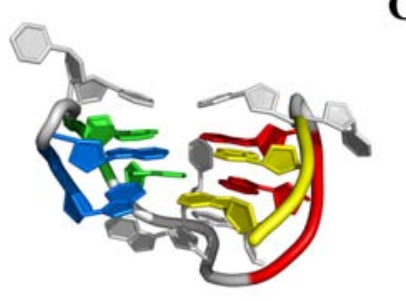

C)

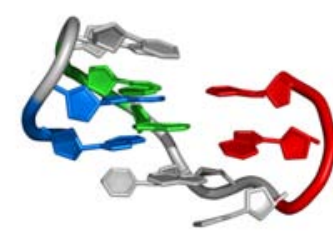

ก $90^{\circ}$

D)

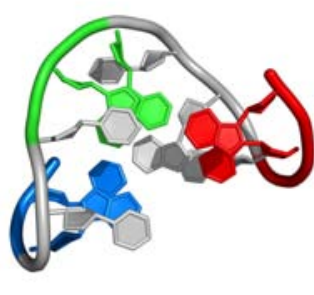

Figure 2. Representation of the 3D structure of the TBA DNA Gquadruplex (A, B) (PDB ID: 1QDF) and of the G-triplex conformation of the 11-mer-3'-t-TBA fragment predicted by metadynamics calculation (C, D) (17). The four GG tracts of TBA are displayed with different color: G1-G2 in blue, G5-G6 in green, G10-G11 in red and G14-G15 (for TBA only) in yellow.
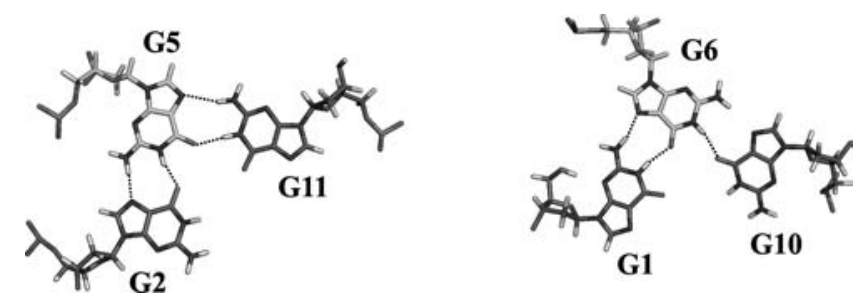

Figure 3. Top view of the two G-triad planes with the experimentally detected hydrogen-bond network.

A well-known issue, encountered in the structure calculation of nucleic acids, is the low proton density and the lack of long-range NOE interactions. Therefore, the quality of experimental structures based on classical restraints such as NOE and J-couplings may not be high enough to permit an analysis at atomic resolution and to solve possible structural ambiguities. Residual dipolar couplings (RDCs) can greatly improve the quality of the calculated structures, providing long-range information and increasing their precision and accuracy (19-21). Moreover, since RDCs depend on the relative orientations of individual bond vectors in the molecule, they can improve the local and global folding also in small DNA/RNA constructs (22). Therefore, we set to solve the fully experimental solution structure of the $3^{\prime}$ TBA-truncated 11-mer oligonucleotide (11-mer$3^{\prime}$-t-TBA) by using both the traditional solution NMR restraints (NOEs and torsional angles) and one-bond RDCs. This is the first experimentally determined structure of a G-triplex oligonucleotide arrangement, and the first entry of this type in the protein data bank (PDB codes: 2MKM and $2 \mathrm{MKO}$ ). The overall folding predicted by metadynamics simulations is confirmed by the experimental structure. However, few differences are observed. 


\section{MATERIALS AND METHODS}

\section{Sample preparation}

All oligonucleotides were synthesized using standard protocol. The oligomers were detached from the support and deprotected by treatment with concentrated aqueous ammonia at $328 \mathrm{~K}$ for $12 \mathrm{~h}$. The combined filtrates and washings were concentrated under reduced pressure, redissolved in $\mathrm{H}_{2} \mathrm{O}$, analyzed and purified by highperformance liquid chromatography on a Nucleogel SAX column (Macherey-Nagel, 1000-8/46); using buffer A: 20 $\mathrm{mM} \mathrm{KH} \mathrm{PO}_{4} / \mathrm{K}_{2} \mathrm{HPO}_{4}$ aqueous solution ( $\mathrm{pH} 7.0$ ), containing 20\% (v/v) $\mathrm{CH}_{3} \mathrm{CN}$; buffer B: $1 \mathrm{M} \mathrm{KCl}, 20 \mathrm{mM}$ $\mathrm{KH}_{2} \mathrm{PO}_{4} / \mathrm{K}_{2} \mathrm{HPO}_{4}$ aqueous solution ( $\mathrm{pH} 7.0$ ), containing $20 \%(\mathrm{v} / \mathrm{v}) \mathrm{CH}_{3} \mathrm{CN}$; a linear gradient from $0 \%$ to $100 \% \mathrm{~B}$ for $30 \mathrm{~min}$ and flow rate $1 \mathrm{ml} / \mathrm{min}$ were used. The fractions of the oligomers were collected and successively desalted by Sep-pak cartridges (C-18). The isolated oligomers proved to be $>99 \%$ pure by NMR.

\section{NMR spectroscopy}

The truncated TBA sequences were characterized, after the thermal annealing, by $1 \mathrm{D}{ }^{1} \mathrm{H}-\mathrm{NMR}$ experiments carried out with a Varian Unity-Inova three-channel 700-MHz spectrometer at 278 and $298 \mathrm{~K}$. Homonuclear and heteronuclear 2D NMR experiments $\left(2 \mathrm{D}{ }^{1} \mathrm{H}-{ }^{1} \mathrm{H}\right.$ COSY, 2D ${ }^{1} \mathrm{H}-{ }^{1} \mathrm{H}$ TOCSY, 2D ${ }^{1} \mathrm{H}-{ }^{1} \mathrm{H}$ NOESY (at mixing times of 100 and $300 \mathrm{~ms}$ ) and JR-HMBC), performed to characterize in detail the construct 5'-GGTGTGGTTGG-3', were also carried out at $298 \mathrm{~K}$ with Bruker spectrometers operating at 500, 600 and $800 \mathrm{MHz}$, equipped with triple resonance cryo-probes.

In the case of 11-mer-3'-t-TBA, all experiments were collected on oligomer samples at the concentration of $0.7-0.8$ $\mathrm{mM}$ in potassium phosphate buffer $(70 \mathrm{mM} \mathrm{KCl}, 10 \mathrm{mM}$ $\mathrm{KH}_{2} \mathrm{PO}_{4}, 0.2 \mathrm{mM}$ EDTA, $\mathrm{pH} 7$ ).

Resonance assignments and 304 proton-proton distance restraints were collected on 11-mer-3'-t-TBA as previously described (17). NMR spectra to gather additional restraints were performed at $274 \mathrm{~K}$ on Bruker spectrometers operating at 500 and $600 \mathrm{MHz}$, equipped with triple-resonance cryo-probes. 2D ${ }^{1} \mathrm{H}-{ }^{1} \mathrm{H}$ E.COSY (23) experiments to determine ${ }^{1} \mathrm{H}-{ }^{1} \mathrm{H}$ three-bond couplings were acquired at 500 $\mathrm{MHz}$. For the determination of the dihedral angles of the backbone, phosphorus-based NMR experiments were collected at $600 \mathrm{MHz}$ on a 11-mer-3'-t-TBA sample dissolved in ${ }^{2} \mathrm{H}_{2} \mathrm{O} .{ }^{31} \mathrm{P}$ chemical shifts were referenced to an external standard of $\mathrm{H}_{3} \mathrm{PO}_{4}$ at $85 \%$ and are reported in Table 1. 2D ${ }^{31} \mathrm{P}_{-}{ }^{1} \mathrm{H}$ HSQC (24) experiments were performed using 1968 scan in the $\mathrm{t} 2$ dimension and $80 \mathrm{t} 1$ increments, whereas three-bond ${ }^{1} \mathrm{H}_{-}{ }^{31} \mathrm{P}$ couplings were obtained from the analysis of a constant-time $2 \mathrm{D}^{31} \mathrm{P}-{ }^{1} \mathrm{H}$ COSY (25) acquired with 2720 scans and 50 increments in the $t 2$ dimension. The delays used for the ${ }^{1} \mathrm{H}_{-}{ }^{31} \mathrm{P}$ polarization transfer in these experiments ranged from 12 to $16 \mathrm{~ms}$.

2D ${ }^{13} \mathrm{C}-{ }^{1} \mathrm{H}$ HSQC spectra, centered on the aliphatic and aromatic regions, were collected on a Bruker spectrometer operating at $800 \mathrm{MHz}$ and $277 \mathrm{~K}$ in natural isotopic abundance, and allowed the assignment of ${ }^{13} \mathrm{C}$ resonances. RDCs $\left(\mathrm{D}_{\mathrm{HC}}\right)$ were measured using the InPhase AntiPhase
Table 1. ${ }^{31} \mathrm{P}$ chemical shifts for $11-\mathrm{mer}-3^{\prime}-\mathrm{t}-\mathrm{TBA}$ in the presence of $80 \mathrm{mM}$ of $\mathrm{K}^{+}$at $274 \mathrm{~K}$.

\begin{tabular}{ll}
\hline Residue & ${ }^{31} \mathrm{P}(\mathrm{ppm})$ \\
\hline G1 & -0.622 \\
G2 & -2.152 \\
T3 & -1.576 \\
T4 & -0.538 \\
G5 & -0.901 \\
G6 & -1.146 \\
T7 & -1.376 \\
T9 & -0.029 \\
G10 & -0.862 \\
\hline
\end{tabular}

The chemical shifts have been referenced to a standard of $85 \% \mathrm{H}_{3} \mathrm{PO}_{4}$ in $\mathrm{H}_{2} \mathrm{O}$ at $298 \mathrm{~K}$. The guanines in the syn conformation for the glycosidic torsion angle have been highlighted in bold.

(IPAP) method (26) from non-decoupled 2D ${ }^{1} \mathrm{H}_{-}{ }^{13} \mathrm{C}$ HSQC spectra in the direct dimension. These spectra were recorded in $100 \%{ }^{2} \mathrm{H}_{2} \mathrm{O}$, phosphate buffered solution of 11-mer- $3^{\prime}-$ t-TBA on a Bruker spectrometer operating at $950 \mathrm{MHz}$ equipped with a cryo-probe. The RDCs were calculated as the difference in the splitting of the one-bond ${ }^{1} \mathrm{H}_{-}{ }^{13} \mathrm{C}$ couplings in partially aligned and isotropic samples. Two different alignment media have been used to determine RDCs in G-triplex: (i) bicelles, containing C8E5 glycol and noctanol $(r=0.87)$, at a concentration of C8E5/water equal to $5 \% \mathrm{w} / \mathrm{v}(27)$; (ii) filamentous phages Pf1 $(28,29)$ provided by ASLA biotech at the concentration of $18 \mathrm{mg} / \mathrm{ml}$. For both the isotropic and anisotropic samples, one experiment with the INEPT (Insensitive Nuclei Enanched by Polarization) delay matching the sugar $\mathrm{J}_{\mathrm{CH}}$-coupling $(1 / 4 \mathrm{~J}=1.72$ $\mathrm{ms})$ and a second experiment matching the aromatic $\mathrm{J}_{\mathrm{CH}^{-}}$ coupling $(1 / 4 \mathrm{~J}=1.51 \mathrm{~ms})$ were collected at $950 \mathrm{MHz}$ and $277 \mathrm{~K}$. For each set, $1741\left({ }^{1} \mathrm{H}\right), 448$ and $256\left({ }^{13} \mathrm{C}\right)$ complex points were recorded for spectral widths of $11433 \mathrm{~Hz}\left({ }^{1} \mathrm{H}\right)$ and $20310 \mathrm{~Hz}\left({ }^{13} \mathrm{C}\right)$ for the aliphatic and $3345 \mathrm{~Hz}\left({ }^{13} \mathrm{C}\right)$ for the aromatic spectrum, respectively.

A total of 21 and $14{ }^{13} \mathrm{C}-{ }^{1} \mathrm{H}$ RDCs were collected from the spectra acquired in the presence of bicelles and filamentous phages Pf1, respectively (see Supplementary Table S1 in supplementary data).

All spectra were processed with the Bruker TOPSPIN software packages and analyzed by the program CARA (Computer Aided Resonance Assignment, ETH Zurich) (30).

\section{Circular dichroism spectroscopy}

CD spectroscopy has been used to further investigate the structure adopted by the truncated TBA sequences in solution. Oligonucleotide samples were prepared by using the same buffer solution used for NMR experiments. CD spectra and CD melting curves of oligonucleotide samples were recorded by using a Jasco J-715 spectropolarimeter equipped with a Jasco JPT-423-S temperature controller using 1-mm path-length cuvettes. CD spectra were averaged over three scans at $273 \mathrm{~K}$, which were recorded in the wavelength range of $200-360 \mathrm{~nm}$ at a scan rate of $100 \mathrm{~nm} / \mathrm{min}$ with a response time of $1 \mathrm{~s}$ and a bandwidth of $2 \mathrm{~nm}$. Buffer 
baseline was subtracted from each spectrum. The DNA concentration (as single strand) was $40 \mu \mathrm{M}$. CD melting and annealing curves were recorded as a function of temperature in the range $273-363 \mathrm{~K}$ with a scan rate of $0.5 \mathrm{~K} / \mathrm{min}$ by following $\mathrm{CD}$ signal changes at the wavelength corresponding to maximum absorbance variation upon folding of each sample.

\section{Structure calculations}

The structure of 11-mer-3'-t-TBA was calculated including the experimental NMR restraints in the web service XPLOR-NIH running on the weNMR community portal (https://www.wenmr.eu/) (31). The distances between nonexchangeable protons were estimated based on the NOE cross-peak volumes at a mixing time of $100 \mathrm{~ms}$ using the distance $\mathrm{H}^{\prime}-\mathrm{H} 2^{\prime}(1.85 \AA)$ as reference for the volume calibration. Exchangeable proton-based restraints were obtained from 2D ${ }^{1} \mathrm{H}-{ }^{1} \mathrm{H}$ NOESY spectra acquired with a mixing time of $300 \mathrm{~ms}$. The upper distance limits for all the restraints derived from these spectra were all set to $6.50 \AA$ to take into account possible spin diffusion effects. A total of 304 NOEs restraints were used in the calculations (188 intraresidue, 67 sequential and 49 interesidue) (Supplementary Table S2). Atoms participating in hydrogen bonds in the G-triad planes were restrained with upper and lower distance limits corresponding to ideal hydrogen bonds. For each individual hydrogen bond, heavy atomheavy atom and heavy atom-proton distance restraints have been imposed (Supplementary Table S3). A total of 67 dihedral restraints were implemented from the analysis of the intraresidue NOEs, the ${ }^{1} \mathrm{H}_{-}{ }^{31} \mathrm{P}$ couplings, and phosphorus chemical shifts (Supplementary Table S4). In XPLOR$\mathrm{NIH}$, a random conformation was first generated for the single-stranded 11-mer-3'-t-TBA sequence and then used to produce a family of 400 structures. The experimentally determined distance restraints, G-triad hydrogen-bonding restraints and dihedral angles were included in structure calculations. All ambiguous distance restraints were specified with the SUM averaging option in XPLOR-NIH. The force constants were scaled to $50 \mathrm{kcal} \mathrm{mol}^{-1} \AA^{-2}$ for NOE and hydrogen-bond distance restraints. The force constants of dihedral angle restraints were $200 \mathrm{kcal} \mathrm{mol}^{-1} \mathrm{rad}^{-2}$ in the simulated annealing protocol.

The 400 structures calculated using the NOEs and dihedral angles restraints were refined against the two sets of RDCs, introduced simultaneously (32). A harmonic potential with a flat bottom was used as the dipolar coupling energy term (33) and the RDC potential was turned on only in the cooling stage of the simulated annealing protocol. The initial tensors of the two set of RDCs were estimated with the program FANTALIN implemented in XPLOR-NIH $(34,35)$. The starting structures were either the model of 11mer-3'-t-TBA predicted by metadynamics calculations (17) or the initial experimental structure obtained without the inclusion of RDC. In both cases the subsequent calculations converged to virtually the same RDC tensors. At the first stage of refinement protocol with RDCs, during the tensor convergence, the weight of these restraints was kept at 0.1 $\mathrm{kcal} \mathrm{mol}^{-1} \mathrm{~Hz}^{-2}$. The final axial and rhombic parameters of the tensors were found to converge at the value of $0.023 \times$
$10^{-32}$ and $-0.012 \times 10^{-32} \mathrm{~m}^{3}$, respectively, for the bicelles, while for the filamentous phages the two parameters were found to converge at the value of $0.023 \times 10^{-32}$ and -0.010 $\times 10^{-32} \mathrm{~m}^{3}$, respectively (see Table 2 ). These values have been used in the final structure calculation protocol, where the weight of RDCs was increased to $1 \mathrm{kcal} \mathrm{mol}^{-1} \mathrm{~Hz}^{-2}$. As expected, the orientation of the tensor axes in bicelles and phages is sizable different (Supplementary Figure S1).

\section{Restrained molecular dynamics calculation and structure val- idation}

The 10 lowest energy structures were then subjected to restrained molecular dynamics (rMD) using the AMBER 12.0 (36) package available through the WeNMR web portal (http://py-enmr.cerm.unifi.it/access/index). The ff99sb force field and specific parameters for nucleic acids (DNA_CI.lib) were used in the AMBER calculations (37). NOE and H-bond restraints were applied with force constants of $20 \mathrm{kcal} \mathrm{mol}^{-1} \AA^{-2}$, while sugar and backbone dihedral angles restraints were used with force constants of $200 \mathrm{kcal} \mathrm{mol}^{-1} \mathrm{rad}^{-2}$. RDCs restraints were applied with a force constant of $0.3 \mathrm{kcal} \mathrm{mol}^{-1} \mathrm{~Hz}^{-2}$. The DNA was placed in a box of TIP3P water, with $10 \AA$ distance from the end of the box. Conversely, the reciprocal orientation of guanines forming the G-triads and the presence of G-triad planes was determined only by experimental restraints and not imposed (38). The structures were initially minimized by fixing the DNA atoms and leaving free the TIP3P waters. Then, a second minimization step was performed without any spatial restraints and using only NMR restraints. The rMD calculations were performed using a simulated annealing protocol, all structures were warmed up from 0 to $600 \mathrm{~K}$ in $10 \mathrm{ps}$, maintained at $600 \mathrm{~K}$ for $15 \mathrm{ps}$, and then cooled to $0 \mathrm{~K}$ in $10 \mathrm{ps}$. A time step of $1.0 \mathrm{fs}$ and a cutoff value of $15 \AA$ were used for the evaluation of non-bonded interactions. The final structures were minimized using the steepest descent and conjugate gradient methods. The same protocol was used also to minimize the structures bearing a potassium ion in the cavity between the two G-triad planes. The potassium ion was placed manually in the cavity of the empty G-triplex structures previously calculated with XPLOR-NIH by using the program PYMOL. Distances between the potassium ion and the carbonyl oxygen of the guanines forming the G-triads were restrained according to the experimental values observed in the quadruplex structures (39).

Structural parameters were evaluated using X3DNA (40) and quality assessments were made with Molprobity (41).

\section{Data deposition}

The coordinates for the G-triplex (5'-GGTTGGTGTGG$3^{\prime}$ ) have been deposited in the Protein Data Bank (accession codes $2 \mathrm{MKM}$ and $2 \mathrm{MKO}$ ).

\section{RESULTS}

\section{Analysis of the truncated TBA sequences}

The removal of a single guanine at the $3^{\prime}$ end of TBA to provide the strand 5'-GGTTGGTGTGGTTG-3' already 
Table 2. Starting and final converged tensor parameters for the two external orienting media.

\begin{tabular}{|c|c|c|c|c|}
\hline \multicolumn{5}{|c|}{ A) Bicelle } \\
\hline & Starting values & Final values & Starting values & Final values \\
\hline$\Delta \chi_{\mathrm{ax}}$ & $0.011 \times 10^{-32} \mathrm{~m}^{3}$ & $0.023 \times 10^{-32} \mathrm{~m}^{3}$ & $0.017 \times 10^{-32} \mathrm{~m}^{3}$ & $0.023 \times 10^{-32} \mathrm{~m}^{3}$ \\
\hline$\Delta \chi_{\mathrm{rh}}$ & $-0.004 \times 10^{-32} \mathrm{~m}^{3}$ & $-0.012 \times 10^{-32} \mathrm{~m}^{3}$ & $-0.010 \times 10^{-32} \mathrm{~m}^{3}$ & $-0.012 \times 10^{-32} \mathrm{~m}^{3}$ \\
\hline \multicolumn{5}{|c|}{ B) Filamentous phages, Pf1 } \\
\hline & Starting values & Final values & Starting values & Final values \\
\hline$\Delta \chi_{\mathrm{ax}}$ & $0.016 \times 10^{-32} \mathrm{~m}^{3}$ & $0.023 \times 10^{-32} \mathrm{~m}^{3}$ & $0.021 \times 10^{-32} \mathrm{~m}^{3}$ & $0.022 \times 10^{-32} \mathrm{~m}^{3}$ \\
\hline$\Delta \chi_{\mathrm{rh}}$ & $-0.010 \times 10^{-32} \mathrm{~m}^{3}$ & $-0.010 \times 10^{-32} \mathrm{~m}^{3}$ & $-0.010 \times 10^{-32} \mathrm{~m}^{3}$ & $-0.010 \times 10^{-32} \mathrm{~m}^{3}$ \\
\hline
\end{tabular}

The initial tensor parameters were obtained by either using the metadynamics-predicted model or the experimental structure calculated from the NOEs and dihedral angles. The member of the family closest to the mean structure was used as experimental structure in tensor analysis.

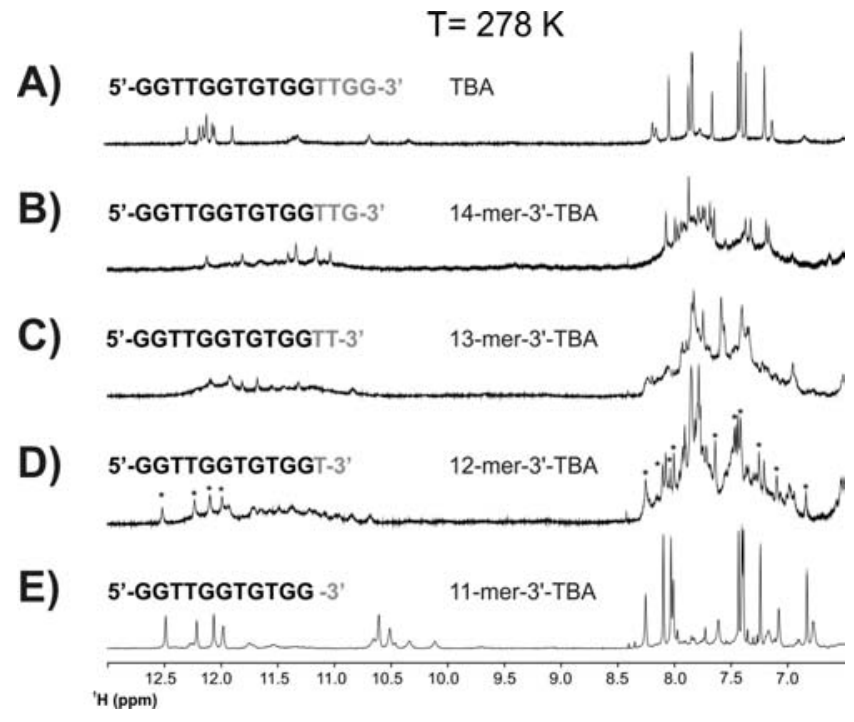

Figure 4. $1 \mathrm{D}^{1} \mathrm{H}$ NMR spectrum of TBA (panel A), and of its truncated sequences at the $3^{\prime}$ end (panels B-E) collected at $278 \mathrm{~K}$.

destabilizes the G-quadruplex structure. The several aromatic signals observed in the $1 \mathrm{D}{ }^{1} \mathrm{H}$ NMR spectra indicate a conformational heterogeneity of the construct, with six weak signals corresponding to imino protons still present in the region between 12.5 and $11 \mathrm{ppm}$ (Figure 4B). The removal of a second guanine at the $3^{\prime}$ end seems to increase the conformational fluctuations and the heterogeneity of the construct with the predominance of broad signals in the aromatic and imino protons regions (Figure 4C). Conversely, the signals of the G-triplex conformation are already unequivocally present in the spectra of the construct lacking the last three bases at the $3^{\prime}$ end (starred signals in Figure 4D). Although conformational heterogeneity is still present in this construct, a slow exchange equilibrium shifted toward the G-triplex folding begins to be present in solution. Figure 4E shows the already described 11-mer-3't-TBA (17). Collectively, these results underline the stabilizing effects of the two guanines at the $3^{\prime}$ end on the Gquadruplex folding, but more important they show the critical role of the two thymines forming the loop in the TBA,

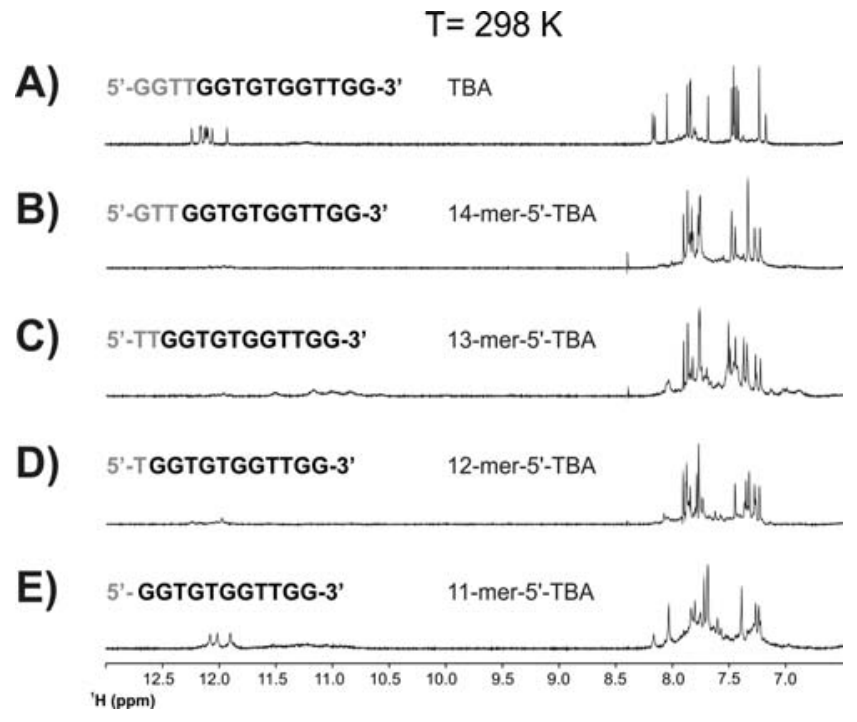

Figure 5. 1D ${ }^{1} \mathrm{H}$ NMR spectrum of TBA (panel A), and of its truncated sequences at the $5^{\prime}$ end (panels B-E) collected at $298 \mathrm{~K}$.

and here observed to destabilize the G-triplex conformation. The destabilizing effect of the truncations at the $5^{\prime}$ end on the G-quadruplex structure of TBA is immediately apparent from the $1 \mathrm{D}{ }^{1} \mathrm{H}$ NMR spectra of the corresponding constructs (Figure 5B-D). More importantly, the construct obtained by the removal of the four bases at the $5^{\prime}$ end of TBA (Figure 5E), which would correspond to a possible structural analog of 11-mer-3'-t-TBA, is affected by conformational heterogeneity. 2D spectra suggest the presence of two distinct species. The full NMR characterization of the latter to prove or disprove the possible presence of G-triplex folding will be the subject of a subsequent work (see Supplementary Data).

The CD spectrum of 11-mer-3'-t-TBA shows two positive bands at $289 \mathrm{~nm}$ and $253 \mathrm{~nm}$, respectively, and a negative band at $265 \mathrm{~nm}$ (17). This spectrum resembles that of TBA (with two positive bands at 294 and $247 \mathrm{~nm}$, respectively, and a negative band at $268 \mathrm{~nm}$ ). These $\mathrm{CD}$ profiles are indicative of a homopolar stacking of the nucleobases (42). However, in the 11-mer-3'-t-TBA construct both posi- 
tive and negative bands are slightly shifted, thus suggesting that the stacking of the bases is not identical to that present in TBA.

The CD melting profile of 11 -mer- $3^{\prime}$-t-TBA shows almost superimposable heating and cooling curves (17), therefore the melting profile is reversible, with no significant hysteresis. For the 11-mer-3'-t-TBA the analysis of the CD melting profiles have provided a melting temperature, $T_{\mathrm{m}}$, of $307( \pm 1) \mathrm{K}$, and a van't Hoff enthalpy change $\left(\Delta H_{\mathrm{vH}}^{\circ}\right)$ of $145( \pm 15) \mathrm{kJ} \mathrm{mol}^{-1}$. Furthermore, DSC experiments were carried out to characterize the denaturation thermodynamics of 11-mer-3'-t-TBA with a model-independent analysis method (17). The integration of the denaturation peaks provided a $\Delta H^{\circ}$ cal of $135( \pm 5) \mathrm{kJmol}^{-1}$, which is almost identical to the van't Hoff enthalpy calculated from DSC curves, and very close to that calculated from $C D$. This indicates that the transition of $11-$ mer- $3^{\prime}-\mathrm{t}-\mathrm{TBA}$ is a two-state process.

To investigate the conformational heterogeneity of the truncated sequences at $3^{\prime}$ and $5^{\prime}$ ends of TBA, we have also acquired CD spectra, and CD melting and annealing curves, for all the constructs. The analysis shows that most of the melting and annealing CD experiments are characterized by hysteresis. Moreover, the CD spectra, before and after denaturation, are not superimposable (see Supplementary Data section, Supplementary Figures S12 and S13). This indicates that most truncated forms are characterized by conformational heterogeneity. These observations are in good agreement with the $1 \mathrm{D}{ }^{1} \mathrm{H}-\mathrm{NMR}$ experiments. Only in the case of 11-mer-5'-t-TBA the CD spectrum, characterized by two positive bands at 292 and $244 \mathrm{~nm}$, respectively, and a negative band at $263 \mathrm{~nm}$, indicate the prevalence of a main conformation. As reported for the 11-mer$3^{\prime}$-t-TBA, also for the main conformation of the 11-mer- $5^{\prime}$ t-TBA, the small differences observed in the CD spectrum with respect to the TBA construct can reasonably be explained by a slightly different arrangement of the stacking between the two G-triads.

\section{Glycosidic torsion angle determination and sugar and back- bone conformations of the 11-mer-3'-t-TBA}

The proton resonance assignment has been previously reported (17) and it was performed through the analysis of 2D ${ }^{1} \mathrm{H}_{-}{ }^{1} \mathrm{H}$ NOESY, 2D ${ }^{1} \mathrm{H}_{-}{ }^{1} \mathrm{H}$ TOCSY, 2D ${ }^{1} \mathrm{H}_{-}{ }^{1} \mathrm{H}$ DQF COSY and JR-HMBC NMR spectra.

Structural information on 11-mer-3'-t-TBA could be retrieved by both $2 \mathrm{D}{ }^{1} \mathrm{H}_{-}{ }^{1} \mathrm{H}$ NOESY and $2 \mathrm{D}{ }^{1} \mathrm{H}_{-}{ }^{1} \mathrm{H}$ COSY experiments. In particular, according to the $\mathrm{H} 8-\mathrm{H}^{\prime}$ and $\mathrm{H} 8-\mathrm{H} 2^{\prime} / \mathrm{H} 2^{\prime \prime} \mathrm{NOE}$ relative intensities in the $2 \mathrm{D}{ }^{1} \mathrm{H}-$ ${ }^{1} \mathrm{H}$ NOESY spectrum of 11-mer-3'-t-TBA, three guanine residues $(\mathrm{G} 1, \mathrm{G} 5, \mathrm{G} 10)$ were classified in the syn conformation, and the four other guanine residues (G2, G6, G8, $\mathrm{G} 11$ ) in the anti conformation. The glycosidic torsion angle $\chi$ was thus restricted in the structure calculation to $60^{\circ} \pm 40^{\circ}$ and to $240^{\circ} \pm 40^{\circ}$ for the two different classes of residues, respectively. The range $240^{\circ} \pm 70^{\circ}$ was used to restraint the glycosidic torsion angle $\chi$ of the thymine bases belonging to the loops.

The prevalent conformation of the sugar was determined from the analysis of the 2D ${ }^{1} \mathrm{H}_{-}{ }^{1} \mathrm{H}$ DQF-COSY and $2 \mathrm{D}^{1} \mathrm{H}-$
${ }^{1} \mathrm{H}$ E.COSY spectra. In the 2D ${ }^{1} \mathrm{H}_{-}{ }^{1} \mathrm{H}$ DQF-COSY spectrum strong $\mathrm{H}^{\prime}-\mathrm{H} 2^{\prime}$ cross-peaks are observed for $\mathrm{G} 2$, T3, T4, G5, G6, T7, T9, G10. Conversely, stronger H1'-H2' cross-peaks are detected for G1 and G8. At the same time, G11 displays $\mathrm{H}^{\prime}-\mathrm{H} 2^{\prime}$ and $\mathrm{H}^{\prime}{ }^{\prime}-\mathrm{H} 2^{\prime} 2 \mathrm{D}$ COSY cross-peaks of equal intensity. All the $\mathrm{H}^{\prime}-\mathrm{H} 4^{\prime}$ cross-peaks, except for G11, are missing in this spectrum, due to the very weak coupling between these two protons. These evidences indicate that all nucleotides, but G11, adopt predominantly a Stype, $\mathrm{C} 2$ '-endo, sugar conformation, while G11 possibly interconverts between the $\mathrm{C}^{\prime}$-endo and $\mathrm{C} 2^{\prime}$-endo conformations. As previously observed in the $2 \mathrm{D}^{1} \mathrm{H}-{ }^{1} \mathrm{H}$ DQF-COSY, also in the $2 \mathrm{D}^{1} \mathrm{H}-{ }^{1} \mathrm{H}$ E.COSY spectrum, the cross-peaks related to $\mathrm{H}^{\prime}$ - $-\mathrm{H} 4^{\prime}$ coupling are absent, further suggesting that the two protons are staggered by $90^{\circ}$, as it occurs in the $\mathrm{C} 2^{\prime}$-endo conformation. The ${ }^{3} \mathrm{~J}$ couplings evaluated from the $2 \mathrm{D}{ }^{1} \mathrm{H}-{ }^{1} \mathrm{H}$ E.COSY spectrum in the $\mathrm{H} 1^{\prime}-\mathrm{H} 2^{\prime} / 2^{\prime}$ region are in agreement with a predominance of the $\mathrm{S}$-type sugar conformation $\left({ }^{3} \mathrm{~J}_{1^{\prime} 2^{\prime}}\right.$ is in the range between 7 and $\left.11 \mathrm{~Hz}\right)$. Therefore, in order to maintain the $\mathrm{C} 2$ '-endo conformation of the sugars in XPLOR-NIH calculation, the dihedral angles $\nu_{1}$ and $\nu_{2}$ for all nucleotides, but G11, were restricted to $30^{\circ} \pm 5^{\circ}$ and $-27.5^{\circ} \pm 5^{\circ}$, respectively. Furthermore, a value of $-90^{\circ} \pm 5^{\circ}$ was imposed to the pseudo-dihedral angle $\mathrm{H}^{\prime}-\mathrm{C}^{\prime}{ }^{\prime}-\mathrm{C}^{\prime}-\mathrm{H}^{\prime}$ to maintain the $\mathrm{H} 3^{\prime}$ and $\mathrm{H}^{\prime}$ ' protons turned by $90^{\circ}$, as indicated by the experimental data. In AMBER calculations, the range for the pseudo-rotation phase angle $P$ typical of the $\mathrm{C} 2^{\prime}$-endo conformation $\left(171^{\circ}\right.$ $\pm 9^{\circ}$ ) was directly imposed.

The values of the backbone dihedral angles have been determined (i) from the analysis of the relative peak intensities of the $2 \mathrm{D}{ }^{1} \mathrm{H}-{ }^{1} \mathrm{H}$ NOESY spectrum, (ii) from the qualitative analysis of the ${ }^{31} \mathrm{P}-\mathrm{NMR}$ spectra and (iii) from the evaluation of the ${ }^{31} \mathrm{P}-{ }^{1} \mathrm{H}$ coupling constants.

The ranges for the $\gamma$ backbone torsion angles have been derived from the analysis of the intensity of the NOESY cross-peaks between aromatic and sugar protons and sugar-sugar protons (43). No cross-peaks or very weak cross-peaks were found from $\mathrm{H} 6 / \mathrm{H} 8$ to any $\mathrm{H}^{\prime} / \mathrm{H}^{\prime \prime}$ protons; moreover, $\mathrm{H} 2^{\prime}-\mathrm{H} 5^{\prime} / \mathrm{H} 5^{\prime}$ cross-peaks are weaker than $\mathrm{H} 2^{\prime}-\mathrm{H} 1^{\prime}$ cross-peaks for all the residues but for $\mathrm{G} 5$ and $\mathrm{G} 10$, where the relative intensity of the two cross-peaks is similar. These spectral features allowed us to restrict the backbone $\gamma$ angle (of all residues but G5 and G10) in the range $60^{\circ} \pm$ $40^{\circ}$, which results in a swinging of the geminal $\mathrm{H} 5^{\prime} / \mathrm{H} 5^{\prime}$ protons away from the core of the DNA helix into the solvent and far from the aromatic and $\mathrm{H} 2^{\prime}$ protons (43). These findings are further supported by the phosphorus-based NMR experiments.

The 2D ${ }^{31} \mathrm{P}-{ }^{1} \mathrm{H}$ HSQC (24) and $2 \mathrm{D}{ }^{31} \mathrm{P}-{ }^{1} \mathrm{H}$ COSY (25) spectra (Figure 6) enabled us to assign the phosphorus resonances of 11-mer-3'-t-TBA. The analysis of the chemical shifts shows that most of the ${ }^{31} \mathrm{P}$ resonances are clustered in the region $-1 \pm 0.5 \mathrm{ppm}$, as reported for regular righthanded B-DNAs. A second group of signals corresponding to T3, T4 and G10 fall outside this region (Table 1). These experimental evidences support the absence of a helical structure for the T3-T4 region and suggest a higher flexibility of this loop. Also the non-canonical value of the ${ }^{31} \mathrm{P}$ chemical shift of G10 could be explained by an increased 

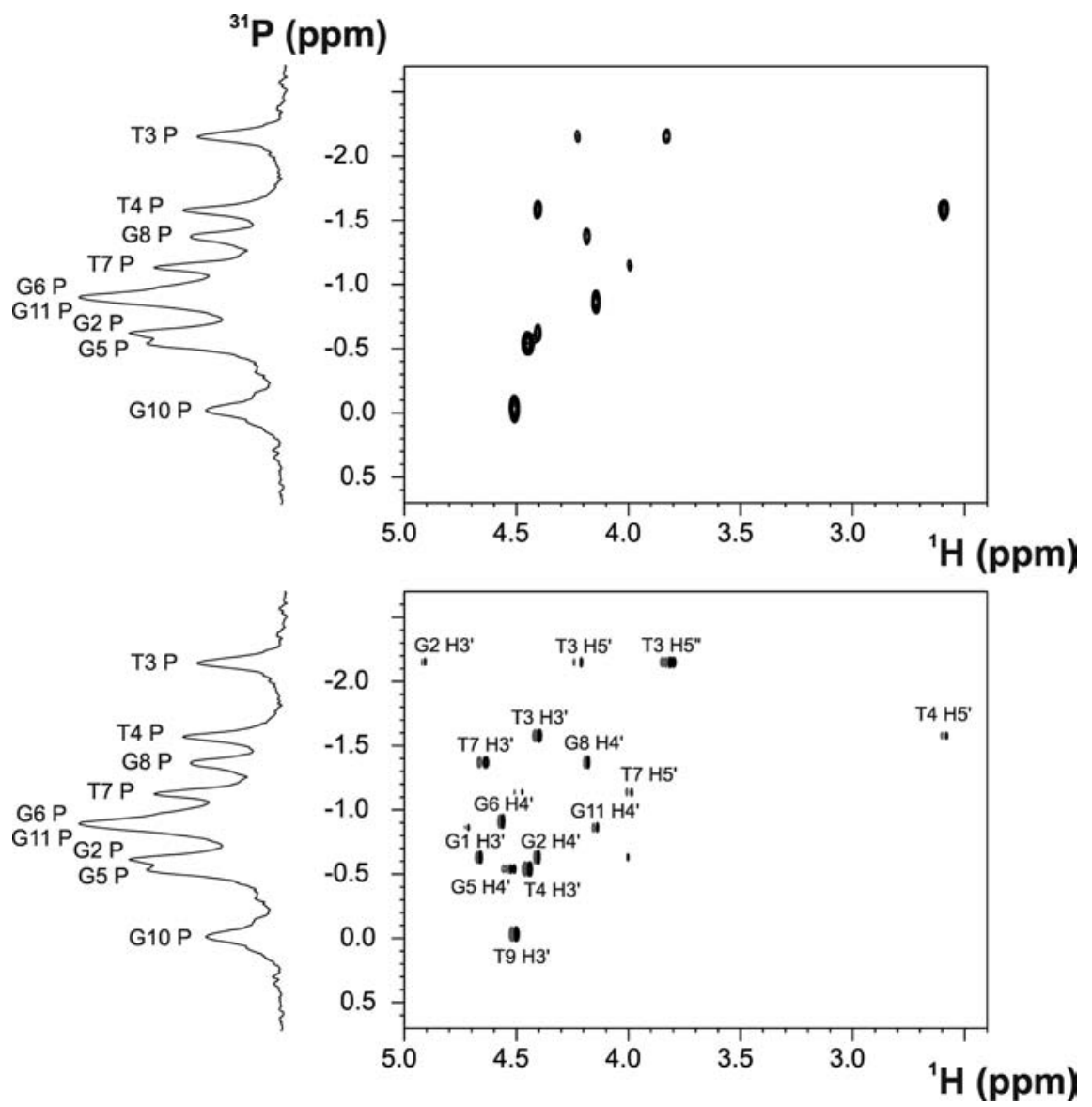

Figure 6. ${ }^{31} \mathrm{P}-{ }^{1} \mathrm{H}$ HSQC (top) and ${ }^{31} \mathrm{P}_{-}{ }^{1} \mathrm{H}$ COSY (bottom) of 11 -mer- $3^{\prime}$-t-TBA at $600 \mathrm{MHz}$ (proton Larmor frequency), and $274 \mathrm{~K}$.

flexibility due to the lack of the fourth strand in the Gtriplex structure.

Additional information on the backbone torsion angles were obtained from the analysis of the $2 \mathrm{D}^{31} \mathrm{P}-{ }^{1} \mathrm{H}$ HSQC and $2 \mathrm{D}{ }^{31} \mathrm{P}_{-}{ }^{1} \mathrm{H}$ COSY spectra. In the $2 \mathrm{D}{ }^{31} \mathrm{P}_{-}{ }^{1} \mathrm{H}$ HSQC some ${ }^{31} \mathrm{P}$ nuclei provide strong cross-peaks with either the $\mathrm{H} 3^{\prime}$ sugar proton of the $5^{\prime}$-adjacent nucleotide, or with the $\mathrm{H} 4^{\prime}$ of the $3^{\prime}$-adjacent nucleotide. Therefore, the backbone torsion angle $\gamma$ was restrained according to the presence or the absence of the cross-peak between ${ }^{31} \mathrm{P}$ and $\mathrm{H}^{\prime}$, as detailed below. It is also interesting to note that all the nucleotides with syn conformation for the glycosidic torsion angle exhibit the cross-peak between ${ }^{31} \mathrm{P}$ and $\mathrm{H}^{\prime}$, while those in the anti conformation show a cross-peak between ${ }^{31} \mathrm{P}$ and $\mathrm{H} 4{ }^{\prime}$, although the different cross-peak pattern is a consequence of both sugar and backbone conformational preferences. More in detail, a strong cross-peak between the phosphorus and the $\mathrm{H}^{\prime}{ }^{\prime}$ sugar proton of the previous nucleotide is observed for G5 and G10, while a crosspeak between the phosphorus and the $\mathrm{H} 4^{\prime}$ proton of its own sugar is present for G2, G6 and G11. Moreover, in the $2 \mathrm{D}^{31} \mathrm{P}-{ }^{1} \mathrm{H}$ HSQC, both ${ }^{31} \mathrm{P}$ cross-peaks with the adjacent sugar protons $\mathrm{H}^{\prime}$ and $\mathrm{H}^{\prime \prime}$ are absent for all the guanines. Conversely, in the case of T3, T4 and T7, correlations among ${ }^{31} \mathrm{P}$ with either both $\mathrm{H}^{\prime}-\mathrm{H} 5^{\prime}$ or only $\mathrm{H}^{\prime}$ are present. The cross-peak corresponding to the phosphorus of T9 could not be assigned and therefore no angular re- straint was applied to $\mathrm{T} 9$ in structure calculations. The 2D ${ }^{31} \mathrm{P}_{-}{ }^{1} \mathrm{H}$ COSY was acquired with a smaller coupling constant for the ${ }^{1} \mathrm{H}-{ }^{31} \mathrm{P}$ polarization transfer (16 instead of 20 $\mathrm{Hz}$ used in the $2 \mathrm{D}^{31} \mathrm{P}_{-}{ }^{1} \mathrm{H}$ HSQC). In this case, besides the correlations observed in the $2 \mathrm{D}^{31} \mathrm{P}-{ }^{1} \mathrm{H}$ HSQC, the crosspeaks between the phosphorus atoms of all the nucleotides with the $\mathrm{H}^{\prime}$ ' sugar-proton of the antecedent residues were identified. Few weak cross-peaks between the phosphorus and the proximal $\mathrm{H}^{\prime} / \mathrm{H} 5^{\prime}$ protons (i.e. only in the case of G2 and G6) were detected. Although ${ }^{4} \mathbf{J}_{\mathrm{H} 4^{\prime}-\mathrm{P},}{ }^{3} \mathbf{J}_{\mathrm{H} 5^{\prime}-\mathrm{P}}$ and ${ }^{3} \mathrm{~J}_{\mathrm{H} 5^{\prime \prime}-\mathrm{P}}$ cannot be accurately measured, the presence or $\mathrm{ab}$ sence of the corresponding cross-peaks provides qualitative information about the conformation of the backbone. In particular, the absence or very weak intensity of both the $\mathrm{H}^{\prime}-\mathrm{P}$ and $\mathrm{H} 5^{\prime}-\mathrm{P}$ signals indicates that the $\beta$ backbone torsion angle is in the canonical trans conformation (44). Therefore, in the structure calculation the $\beta$ torsion angle of the following residues, G2, G5, G6, G8, G10, G11, was restricted to the range $180^{\circ} \pm 50^{\circ}$. Moreover, in the case of the guanines in the anti conformation, the ${ }^{4} \mathbf{J}_{\mathrm{H}^{\prime} \text {-P }}$ coupling constants are large enough to give rise to cross-peaks in the spectrum; this occurs only when $\beta / \gamma$ fall in the trans/g+ region $(44,45)$. The unambiguous presence of all $\mathrm{H} 4$ '-P crosspeaks confirms that for G2, G6 and G11 $\beta$ is in trans and $\gamma$ is $\mathrm{g}+$ conformation. Therefore, also the analysis of the $2 \mathrm{D}$ ${ }^{31} \mathrm{P}-{ }^{1} \mathrm{H}$ spectra, as well as of the NOESY peak intensities, confirmed the range $60^{\circ} \pm 40^{\circ}$ for the $\gamma$ torsion angle. 


\section{Determination of the 11-mer-3'-t-TBA folding topology}

The folding topology of 11 -mer- $3^{\prime}$-t-TBA, from the analysis of characteristic NOEs between imino and $\mathrm{H} 8$ protons, was already proposed to be consistent with an intramolecular G-triplex involving two G-triads, G1:G6:G10 and G2:G5:G11. The presence of the two G-triads is further supported by additional NOEs among the $\mathrm{H} 1$ protons and of the loop protons with the two G-triads (Supplementary Figure S2). The hydrogen-bond directionalities of the two putative G-triads are clockwise and anti-clockwise, respectively (Figure 3). The glycosidic conformations of guanines are syn-anti-syn around the first G-triad and anti-syn-anti around the second G-triad, as it occurs in the corresponding G-quadruplex. These glycosidic conformations are consistent with an antiparallel-stranded G-triplex core, containing three G-tracts (G1-G2, G5-G6, G10-G11) with alternating anti-parallel orientations. As in TBA, the TT and TGT tracts exhibit a peculiar pattern of NOEs and form loops at opposite ends of the G-triplex that span the narrow and the wide grooves, respectively. In the proposed G-triplex fold, the imino protons of G1, G6, G5 and G11 are involved in the formation of Hoogsten-like hydrogen bonds forming the G-triads. Therefore, these protons are the most protected from the exchange with water. On the other hand, imino protons of G2 and G10 are not involved in any hydrogen bond and exchange quickly with the solvent. This picture is consistent with the presence of only four sharp signals in the region of the imino protons of the ${ }^{1} \mathrm{H}$ NMR spectrum (Figure 1).

\section{NMR structure determination and structure statistics of 11- mer-3'-t-TBA}

The ' $a b$ initio' solution structure of 11-mer-3'-t-TBA was calculated with XPLOR-NIH using a simulated annealing protocol, starting from a single-stranded linear DNA structure. All the experimental restraints including distance restraints, glycosidic torsion angles, sugar and backbone conformations, and subsequently RDCs as described in the Materials and Methods section, were used in the calculations. NOE distance restraints were obtained from the analysis of $2 \mathrm{D}{ }^{1} \mathrm{H}-{ }^{1} \mathrm{H}$ NOESY spectra acquired with different mixing times (100 and $300 \mathrm{~ms}$ ). On average 28 NOE-derived distance restraints and six angle restraints per residue were used for the structure calculation (Table 3). Hydrogen-bond restraints between G5-G2, G11-G5, G1-G6 and G6-G10, respectively, were also included in the structure-calculation protocol. These hydrogen bonds were identified by the presence and the typical chemical shifts of the signals corresponding to the imino and amino exchangeable protons of the bases. The presence of three Hoogsteen-like base parings (G5-G2, G11-G5, G1-G6) is also confirmed by the NOEs between the imino protons and the aromatic protons of the interacting bases. Also, the downfield shifts of the $\mathrm{H} 21$ protons for $\mathrm{G} 1, \mathrm{G} 5, \mathrm{G} 11$ agree with the presence of a hydrogen bond involving the amino proton and N7 of the neighboring base. In the case of G6, the formation of the $\mathrm{H}$-bond involving its own imino proton and $\mathrm{O} 6$ of G10 is demonstrated by the presence in the spectra of a sharp peak corresponding to H1 of G6. Conversely, the absence
Table 3. NMR distance, dihedral angles and RDC restraints used in the structure calculations.

NMR distance and dihedral angle restraints

\begin{tabular}{ll}
\hline Distance restraints & 304 \\
Total number of NOEs & 188 \\
Intraresidue $[i=j]$ & 115 \\
Interresidue & 67 \\
Sequential $[|i-j|=1]$ & 49 \\
Non-sequential & 22 \\
Hydrogen bonds & \\
Dihedral restraints & 67 \\
Total number of dihedral angles & 39 \\
Backbone dihedral angles: & 6 \\
$\beta$ & 9 \\
$\gamma$ & 22 \\
$\chi$ & 30 \\
Sugar dihedral angles & \\
Residual dipolar couplings & \\
Bicelles & \\
Aliphatic & 13 \\
Aromatic & 8 \\
Phages Pf1 & \\
Aliphatic & 6 \\
Aromatic & 8 \\
\hline
\end{tabular}

of a downfield shift for the $\mathrm{H} 21$ proton of $\mathrm{G} 6$ and the absence of NOEs of H1 and H21 of G6 with H8 of G10 did not allow us to establish the presence of a hydrogen bond between H21 of G6 and N7 of G10. Therefore, this particular interaction was not implemented as a restraint in the calculation.

The 10 lowest energy structures calculated using only short-range interactions (NOEs, H-bonds and dihedral angles) (Supplementary Figure S3) were selected for further analysis. This family already shows a well-defined structure (Root Mean Square Deviation, RMSD 0.616) and a good local structure statistics with no NOE violation $>0.5 \AA$ and no torsion angle violation $>10^{\circ}$. However, visual inspection of the structures shows that the two G-triad planes are quite distorted in all the members of the family. The nonplanarity of the two G-triads is easily attributable to the lack of experimental restraints. To verify this hypothesis and to improve the quality of the experimental structure, RDCs measured in the presence of two different orienting media (bicelles and filamentous phages Pf1) have been included in the refinement of the G-triplex structure.

The experimental RDCs used in XPLOR-NIH included the $\mathrm{C} 1^{\prime}-\mathrm{H} 1^{\prime}, \mathrm{C} 4^{\prime}-\mathrm{H} 4^{\prime}$ and the aromatic $\mathrm{C}-\mathrm{H}$ groups. The $\mathrm{C} 3^{\prime}-\mathrm{H}^{\prime}, \mathrm{C}^{\prime}-\mathrm{H} 2^{\prime}$ and $\mathrm{C} 2^{\prime}-\mathrm{H} 2^{\prime \prime}$ RDCs have not been considered for the refinement because of their proximity to the signal of water, and the sensitivity to the conformational equilibrium of the deoxyribose ring, respectively. The family of 10 structures, refined with RDC and with the lowest XPLOR-NIH energy function, exhibited again good structural statistics, a good agreement with the experimental RDCs, and also an improved planarity of G-triads. The local and global folding is well defined with an RMSD of 0.537 . This family of 10 structures was minimized in AMBER through a molecular dynamics protocol. The final family of minimized structures (see Figure 7) displays a total RMSD of 0.522 . The structure minimization in AMBER was performed both in the presence and in the absence of 
A)

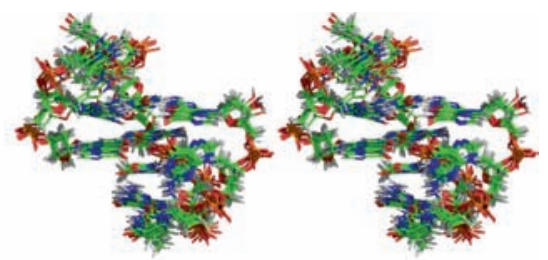

B)

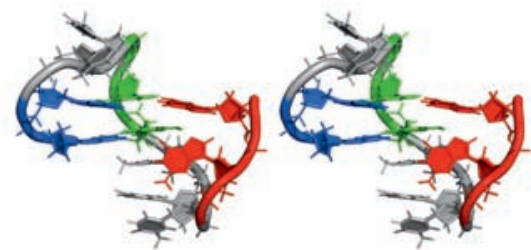

C)

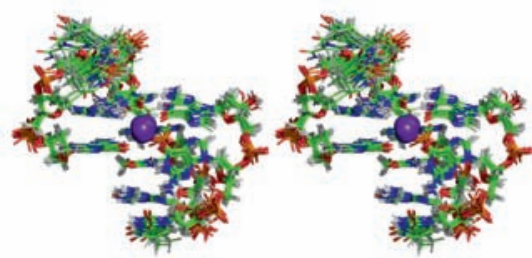

D)

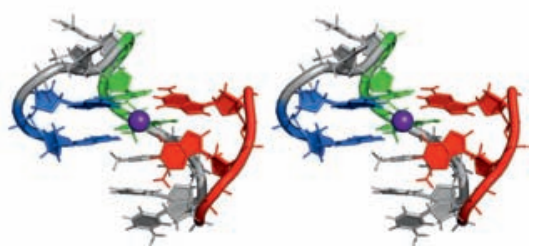

Figure 7. Stereo view representation (crossed eyes) of the family of 10 structures of 11-mer-3'-t-TBA with lowest XPLOR-NIH energy, and after AMBER rMDs (A). The member of the family closest to the mean structure is shown in panel (B) as cartoon. The corresponding family of structures minimized in the presence of the potassium ion is shown in panel (C). The member of the family bearing the potassium ion and closest to the mean structure is shown in panel (D) as cartoon. The potassium ion is shown as a violet sphere.

a potassium ion, placed in the cavity between the two Gtriad planes. The quality of the structural statistics for these two families of minimized structures is similarly good, and is even better when the potassium ion is introduced in the middle of the G-triad planes (Table 4) (Figure 7).

\section{Effect of monovalent cations on G-triplex structure}

The 11-mer-3'-t-TBA construct has been studied also in the presence of monovalent cations other than potassium, such as sodium and ammonium ions. As already observed for TBA, sodium ions do not stabilize the G-triplex structure. In the presence of sodium ions $(80 \mathrm{mM})$ and at room temperature $(298 \mathrm{~K})$, the large number of signals in the aromatic region and the absence of imino protons indicate the presence of unfolded species. Folded species start to appear only at low temperature (i.e. 274 K) (Supplementary Figure S4).

A more detailed analysis has been carried out in the presence of ammonium ions. The titration of the G-triplex with ammonium ions suggests an affinity similar to that reported for the G-quadruplex TBA (16). The addition of ammonium to a solution of 11 -mer-3'-t-TBA increases the amount of a folded species that is present in small per- centage in solution at low temperature, even in the absence of any monovalent cation (Supplementary Figure S5). The established interaction between 11-mer-3'-t-TBA and ammonium is demonstrated by the presence of one signal in the $2 \mathrm{D}{ }^{15} \mathrm{~N}-{ }^{1} \mathrm{H}$ HSQC apart from the signal of bulk ammonium. A second weak cross-peak in $2 \mathrm{D}^{15} \mathrm{~N}-{ }^{1} \mathrm{H}$ HSQC starts to appear by increasing the concentration of ammonium. The analysis of the $2 \mathrm{D}{ }^{1} \mathrm{H}-{ }^{1} \mathrm{H}$ NOESY spectrum of the predominant species present in solution reveals the same pattern of NOEs observed in $\mathrm{K}^{+}-11$-mer-3'-t-TBA, and the same alternation of syn-anti bases. However, even by increasing the concentration of the ammonium ion, not all the unfolded oligonucleotide is converted into the G-triplex conformation (Supplementary Figures S6 and S7).

\section{DISCUSSION}

The pioneering studies of Bock et al. (10) on libraries of guanine-rich oligonucleotides led to the identification of the TBA. The structure of TBA has been extensively characterized by NMR and X-ray crystallography. In TBA, there are only two G-tetrads forming a total of 16 Hoogsteenlike hydrogen bonds (with each guanine involved in four hydrogen bonds) between the imino protons $\mathrm{H} 1$ and oxygen $\mathrm{O} 6$ and between amino protons $\mathrm{H} 21$ and nitrogen N7. In all deposited TBA structures, the two G-tetrad planes are separated by less than $4 \AA(18,46,47)$. TBA is stable over a wide range of temperatures, either in the presence of $\mathrm{K}^{+}$, or $\mathrm{NH}_{4}{ }^{+}$, or of divalent cations such as $\mathrm{Sr}^{2+}$ and $\mathrm{Pb}^{2+}$. Interestingly, multiple species are reported to be present in solution with $\mathrm{Na}^{+}(16)$.

The analysis of the minimized family of structures of 11mer-3'-t-TBA shows that the G-triplex consists of two Gtriads linked with two parallel G-strands interleaved with an anti-parallel G-strand (G1-G2, G5-G6, G10-G11), which are connected together by two side loops (T3-T4, T7-G8T9). The G-triplex of 11-mer-3'-t-TBA maintains a structure that recalls the chair-like, antiparallel structure of TBA also in the absence of the fourth missing strand (Figure 8 ). From the structure of 11-mer-3'-t-TBA it is apparent that one of the bases in the TGT loop (T7) forms stacking interactions with the above G1:G6:G10 plane. As in the G-quadruplex (48), this interaction may have a stabilizing effect. The TGT loop presents many crucial and intense NOEs with the imino protons of the guanines of the neighboring plane. In particular, the methyl group of T7 shows strong correlations with G1-NH, G6-NH and G10-NH. Further NOEs, between the imino proton of G8 and the imino protons of the bases of the G1:G6:G10 plane, have been also identified. Therefore, the TGT loop (in particular bases T7 and G8) is very close to, and quite rigidly held to, the G-stem plane. On the other hand, the TT loop appears more flexible and with a less defined structure. However, some NOEs of G11-HN with T3 and T4 are maintained, indicating that this loop is anyway kept close to the adjacent plane.

The comparison of the TBA structure with that of 11mer-3'-t-TBA shows that the lack of the four bases at the $3^{\prime}$ end in the G-triplex does not cause an extensive structural rearrangement. The stacking bases G1-G2 and G10G11 roughly remain in place and do not form new hydro- 
Table 4. Distance, dihedral angles and RDC statistics of the structure calculations.

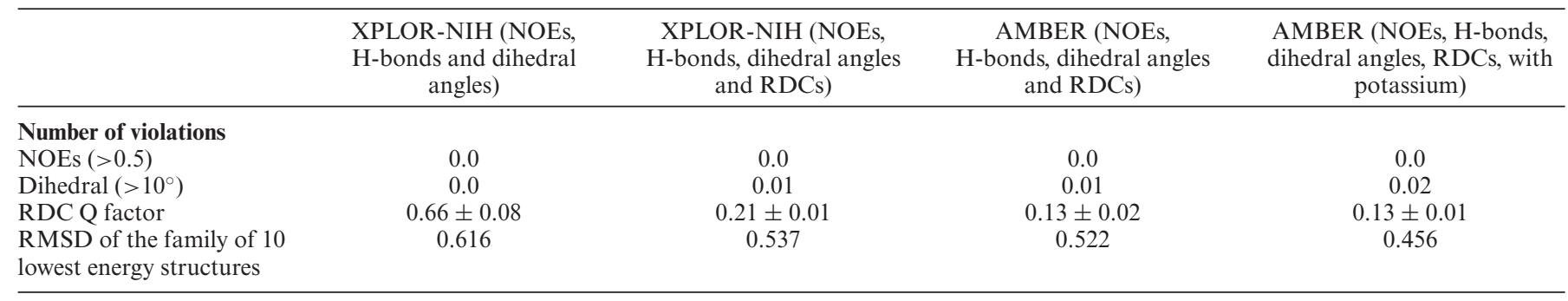

The values are averages for the family of 10 structures. The RMSD are reported with respect to the mean structure.

A)

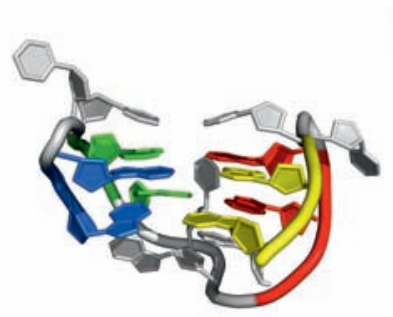

B)

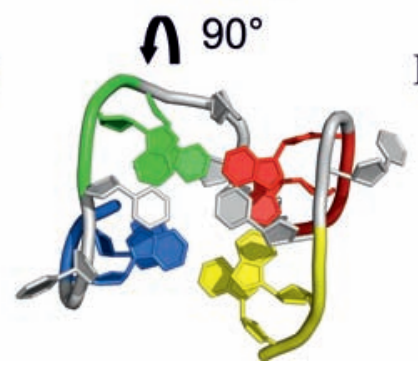

C)

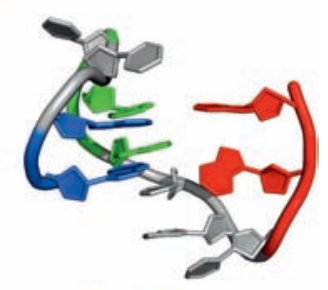

D)

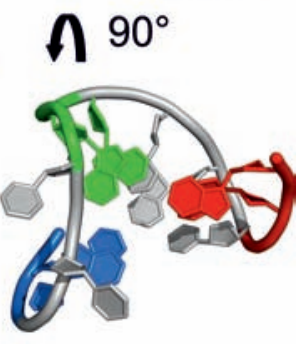

Figure 8. Representation of the 3D structure of the TBA DNA Gquadruplex (A, B) (PDB ID: 1QDF) and of the experimental structure of the 11-mer-3'-t-TBA fragment (C, D) (present work). The four GG tracts of TBA are displayed with different color: G1-G2 in blue, G5-G6 in green, G10-G11 in red and G14-G15 (for TBA only) in yellow.

gen bonds. This is experimentally proven by the loss in the ${ }^{1} \mathrm{H}$ NMR spectra of the signals corresponding to the imino protons of G2 and G10. Such signals can be detected in the spectra only if the imino protons are involved in hydrogen bonds. This finding supports the observation that the Hoogsteen-like hydrogen bonds involving G14 and G15 in TBA are not replaced by new interactions involving G2:G11 and G1:G10. The reduction of Hoogsteen-like hydrogen bonds accounts for the reduced stability of 11-mer- $3^{\prime}-\mathrm{t}-\mathrm{TBA}$ with respect to the TBA structure (Figure 8). Also the similarity between the CD spectrum of TBA and that of 11mer-3'-t-TBA, as well as the shifting of both positive and negative bands, suggests a similar, though not identical, homopolar stacking of the nucleobases $(17,42,49,50)$. For 11mer-3'-t-TBA, the presence of a unimolecular folding was already demonstrated by the CD melting curve and by the DSC analysis (17). In this respect, the possible replacement of G14 and G15 in 11-mer-3'-t-TBA by a GG motif of a different strand has been further investigated. The NMR titrations of the 11-mer-3'-t-TBA with the dinucleotide GG and the oligonucleotide TGGT demonstrate that the two GG motifs do not bind to the G-triplex construct.

Overall, the present experimental ' $a b$ initio' solution structure of 11-mer-3'-t-TBA is largely superimposable to the conformation of the same oligonucleotide stretch as part of the TBA construct along its unfolding pathway predicted by metadynamics (17). The chair-like folding of the backbone is conserved as far as the two G-triads planes are concerned. However, it is interesting to note that the structure of 11-mer-3'-t-TBA in solution shows some differences if compared with the in-silico prediction. This could also be due to intrinsic differences between 11-mer-3'-t-TBA and TBA arising from the absence of the four last bases in the former. The superimposition of the two structures shows a good agreement for the G-triad G2:G5:G11 that is well defined and rather planar also in the experimental structure. Also in the G-triad G1:G6:G10 bases G1 and G6 maintain the same arrangement, while the conformation of G10 could not be defined by the experimental restraints. The aromatic signal of this base is indeed broader than the others, suggesting the presence of a possible conformational exchange involving G10. Moreover, the lack of RDCs and NOEs for G10 has also prevented the identification of an averaged position. Therefore, the obtained orientation for this base is mainly determined by the XPLOR-NIH and AMBER force fields, and thus probably selected because it is energetically more favored in the absence of experimental restraints. In this regard, it should be mentioned that no violations of the experimental restraints were observed when the planarity was imposed to G10 in a calculation performed to evaluate the conformation of this base. On the other hand, it is remarkable that the out-of-plane orientation of G10 is perfectly compatible with the structure of 11-mer-3'-t-TBA calculated by imposing the presence of a potassium ion, where $\mathrm{O} 6$ of G10 appears perfectly poised to coordinate the potassium ion itself.

While the G-triads conformation predicted by metadynamics is largely confirmed by the experiments, structural differences are present in the TGT and TT loops. As described above, in the solution structure of 11-mer-3'-t-TBA, the G-triad plane formed by G1, G6 and G10 is stabilized by $\mathrm{T} 7$, which forms stacking interactions, in particular, with G6. Conversely, in the G-triplex structure predicted by metadynamics, the slightly different arrangement of the TGT loop causes the stacked base T7 to turn away and to be replaced by G8 in its interaction with the same G-triad plane. However, both these residues remain very close to the G-triad plane, suggesting a possible switching in the position of these two residues as a consequence of the absence of the four additional nucleotides at the $3^{\prime}$ end of the 11- 
mer-3'-t-TBA. In the experimental structure of $11-$ mer-3'-tTBA the proximity of G8 to the adjacent G-triad is maintained, although G8 appears slightly translated because of the insertion of T7. Other differences occur at the level of the TT loop. In the structure predicted by metadynamics, T3 and T4 are stacked under the G-triad, although molecular dynamics and metadynamics simulations showed some flexibility of this loop. This arrangement is not present in the experimental structure, where the orientation of these bases is different. In particular, the position of T3 is well determined by many restraints, while that of T4 is less precisely defined, suggesting a more pronounced flexibility of this residue. Therefore, while the loop TGT seems to have a stabilizing effect toward the G-triplex structure, apparently the TT loop does not contribute to stabilize the G-triplex structure and, as in TBA, could be prone to interact with a target (48).

Of particular relevance are the results obtained by the analysis of the NMR spectra collected on the truncated sequences of TBA. In fact, these data reinforce the findings of the metadynamic simulations which indicate the prevalent occurrence of the opening and closing at the $3^{\prime}$ end in the folding/unfolding pathway of TBA. Moreover, the different stability of the truncated sequences of TBA, and their heterogeneity demonstrated by the CD melting curves, underlines the critical effect of the length of TT loops on the stabilization of the G-stem (48). Only in the case of the 11mer-5'-t-TBA, the prevalence of a main conformation has been observed.

This new, detailed, structural information, and the extensive biophysical characterization previously performed on the G-triplex, shed light on the folding mechanism of TBA. The calorimetric and van't Hoff enthalpies of 11-mer-3'-tTBA, previously evaluated from DSC experiments, pointed out that the melting transition occurs in a two-state process, since the value of the two quantities is almost identical (17).

Collectively, our experimental data reinforce the idea that the folding/unfolding process of TBA can proceed as an apparent two-state mechanism. In our conditions the temperature needed to detach G14 and G15 from the Gquadruplex structure is, apparently, high enough to immediately unfold the just formed G-triplex species. Therefore, it is plausible that the folding/unfolding process of the unimolecular G-quadruplex DNA structures occurs through a very fast process, where the G-triplex intermediate is not detectable with the available experimental techniques.

Concerning the effect of the different cations on the folding stability of the G-triplex, our results show that the 11mer-3'-t-TBA largely behaves like the other G-quadruplexforming DNA sequences $(51,52)$. The stabilizing effect observed for $\mathrm{K}^{+}$and $\mathrm{NH}_{4}{ }^{+}$relies on their ionic radius and low dehydration energy. The role of the ionic radius and dehydration energy in the stabilization of the G-quadruplex folding of TBA by $\mathrm{K}^{+}, \mathrm{NH}_{4}{ }^{+}, \mathrm{Rb}^{+}$has been well characterized in the past by $\mathrm{CD}$ spectra and melting profiles (52).

In conclusion, the experimental structure of $11-$ mer- $3^{\prime}-\mathrm{t}$ TBA proves the presence and the stability of the G-triplex folding predicted by metadynamics calculations. The differences between the experimental and predicted structures do not affect the folding of the G-triplex, which is globally maintained. Collectively, the experimental structure here presented is consistent with the possibility that G-triplex folding intermediates may play a role in human genome, and therefore its detailed structural features constitute a useful tool to analyze its interactions with relevant biological partners.

\section{SUPPLEMENTARY DATA}

Supplementary Data are available at NAR Online.

\section{ACKNOWLEDGEMENTS}

Constructive criticisms and several very helpful suggestions by Vladimir Sklenar are gratefully acknowledged. We are also thankful to Janez Plavec for his helpful comments and to Teresa Carlomagno for several suggestions on how to best strengthen the message of the paper.

\section{FUNDING}

MIUR PRIN [PRIN-2012 SK7ASN_001]; Ente Cassa di Risparmio di Firenze, EU Bio-NMR [261863]; ITN pNMR [317127]; EU ESFRI Instruct Core Centre CERM, Italy, AIRC IG 2013 [AIRC \# 14150]; EU WeNMR [261572]. European Union [ERC-2009-AdG-247075]; Gabriele Charitable Foundation. Funding for open access charge: MIUR PRIN [PRIN-2012 SK7ASN_001]; AIRC IG 2013 [AIRC \# 14150].

Conflict of interest statement. None declared.

\section{REFERENCES}

1. Broyde,S., Wang,L., Zhang,L., Rechkoblit,O., Geacintov,N.E. and Patel,D.J. (2008) DNA adduct structure-function relationships: comparing solution with polymerase structures. Chem. Res. Toxicol., 21, 45-52.

2. Phan,A.T. (2010) Human telomeric G-quadruplex: structures of DNA and RNA sequences. FEBS J., 277, 1107-1117.

3. Smith,F.W. (1992) Quadruplex structure of Oxytricha telomeric DNA oligonucleotides. Nature, 356, 164-168.

4. Macaya,R.F., Schultze,P., Smith,F.W., Roe,J.A. and Feigon,J. (1993) Thrombin-binding DNA aptamer forms a unimolecular quadruplex structure in solution. Proc. Natl. Acad. Sci. USA, 90, 3745-3749.

5. Hud,N.V., Schultze,P., Sklenár,V. and Feigon,J. (1999) Binding sites and dynamics of ammonium ions in a telomere repeat DNA quadruplex. J. Mol. Biol., 285, 233-243.

6. Parkinson,G.N., Lee,M.P.H. and Neidle,S. (2002) Crystal structure of parallel quadruplexes from human telomeric DNA. Nature, 417, 876-880.

7. Biffi,G., Tannahill,D., McCafferty,J. and Balasubramanian,S. (2013) Quantitative visualization of DNA G-quadruplex structures in human cells. Nat. Chem., 5, 182-186.

8. Wells,R.D. (2007) Non-B DNA conformations, mutagenesis and disease. Trends Biochem. Sci., 32, 271-278.

9. McLuckie,K.I.E., Di Antonio,M., Zecchini,H., Xian,J., Caldas,C., Krippendorff,B.-F., Tannahill,D., Lowe,C. and Balasubramanian,S. (2013) G-Quadruplex DNA as a Molecular target for induced synthetic lethality in cancer cells. J. Am. Chem. Soc., 135, 9640-9643.

10. Bock,L.C., Griffin,L.C., Latham,J.A., Vermaas,E.H. and Toole,J.J. (1992) Selection of single-stranded DNA molecules that bind and inhibit human thrombin. Nature, 355, 564-566.

11. Crnugelj,M., Hud,N.V. and Plavec,J. (2002) The solution structure of $\mathrm{d}(\mathrm{G}(4) \mathrm{T}(4) \mathrm{G}(3))(2)$ : a bimolecular G-quadruplex with a novel fold. $J$. Mol. Biol., 320, 911-924.

12. Sket,P. and Plavec,J. (2011) Tetramolecular DNA quadruplexes in solution: insights into structural diversity and cation movement. $J$ Am. Chem. Soc., 132, 12724-12732. 
13. Ambrus,A., Chen,D., Dai,J., Bialis,T., Jones,R.A. and Yang,D. (2006) Human telomeric sequence forms a hybrid-type intramolecular G-quadruplex structure with mixed parallel/antiparallel strands in potassium solution. Nucleic Acids Res., 34, 2723-2735.

14. Zhang,Z., Dai,J., Veliath,E., Jones,R.A. and Yang,D. (2010) Structure of a two-G-tetrad intramolecular G-quadruplex formed by a variant human telomeric sequence in $\mathrm{K}+$ solution: insights into the interconversion of human telomeric G-quadruplex structures. Nucleic Acids Res., 38, 1009-1021.

15. Gray,R.D., Trent,J.O. and Chaires,J.B. (2014) Folding and unfolding pathways of the human telomeric G-quadruplex. J. Mol. Biol., 426, 1629-1650

16. Trajkovski,M., Šket,P. and Plavec,J. (2009) Cation localization and movement within DNA thrombin binding aptamer in solution. Org. Biomol. Chem., 7, 4677-4684.

17. Limongelli,V., De Tito,S., Cerofolini,L., Fragai,M., Pagano,B., Trotta,R., Cosconati,S., Marinelli,L., Novellino,E., Bertini,I. et al. (2013) The g-triplex DNA. Angew. Chem. Int. Ed. Engl., 52, 2269-2273.

18. Marathias,V.M., Wang,K.Y., Kumar,S., Pham,T.Q., Swaminathan,S. and Bolton,P.H. (1996) Determination of the number and location of the manganese binding sites of DNA quadruplexes in solution by EPR and NMR in the presence and absence of thrombin. J. Mol. Biol., 260, 378-394.

19. Zídek,L., Padrta,P., Chmelík,J. Jr and Sklenár,V. (2003) Internal consistency of NMR data obtained in partially aligned biomacromolecules. J. Magn. Reson., 162, 385-395.

20. Zídek,L., Stefl,R. and Sklenár,V. (2001) NMR methodology for the study of nucleic acids. Curr. Opin. Struct. Biol., 11, 275-281.

21. Carlomagno,T. (2012) NMR in natural products: understanding conformation, configuration and receptor interactions. Nat. Prod. Rep., 29, 536-554.

22. Vermeulen,A., Zhou,H. and Pardi,A. (2000) Determining DNA global structure and DNA bending by application of NMR residual dipolar couplings. J. Am. Chem. Soc., 122, 9638-9647.

23. Griesinger,C. and Sorensen,O.W. (1987) ECOSY, avance-version (04/01/15);complementary E.COSY program for KcMAX = 3; (number of relevant spins = 3); phase sensitive. J. Magn. Reson., 75, $474-492$.

24. Luy,B. and Marino,J.P. (2001) (1)H-(31)P CPMG-correlated experiments for the assignment of nucleic acids. J. Am. Chem. Soc., 123, 11306-11307.

25. Carlomagno,T., Hennig,M. and Williamson,J.R. (2002) A novel PH-CT-COSY methodology for measuring JPH coupling constants in unlabeled nucleic acids. Application to HIV-2 TAR RNA. $J$. Biomol. NMR, 22, 65-81.

26. Enthart,A., Freudenberger,J.C., Furrer,J., Kessler,H. and Luy,B. (2008) The CLIP/CLAP-HSQC: pure absorptive spectra for the measurement of one-bond couplings. J. Magn. Reson., 192, 314-322.

27. Rückert,M. and Otting,G. (2000) Alignment of biological macromolecules in novel nonionic liquid crystalline media for NMR experiments. J. Am. Chem. Soc., 122, 7793-7797.

28. Hansen,M.R., Hanson,P. and Pardi,A. (2000) Filamentous bacteriophage for aligning RNA, DNA, and proteins for measurement of nuclear magnetic resonance dipolar coupling interactions. Meth. Enzymol., 317, 220-240.

29. Stefl,R., Wu,H., Ravindranathan,S., Sklenár,V. and Feigon,J. (2004) DNA A-tract bending in three dimensions: solving the dA4T4 vs. dT4A4 conundrum. Proc. Natl. Acad. Sci. USA, 101, 1177-1182.

30. Keller,R. (2004) The Computer Aided Resonance Tutorial. CANTINA Verlag, Goldau, Switzerland

31. Schwieters,C.D., Kuszewski,J.J., Tjandra,N. and Clore,G.M. (2003) The Xplor-NIH NMR molecular structure determination package. $J$. Magn. Reson., 160, 65-73.

32. Banci,L., Bertini,I., Cavallaro,G., Giachetti,A., Luchinat,C. and Parigi,G. (2004) Paramagnetism-based restraints for Xplor-NIH. J. Biomol. NMR, 28, 249-261.

33. Tjandra,N., Omichinski,J.G., Gronenborn,A.M., Clore,G.M. and Bax,A. (1997) Use of dipolar $1 \mathrm{H}-15 \mathrm{~N}$ and $1 \mathrm{H}-13 \mathrm{C}$ couplings in the structure determination of magnetically oriented macromolecules in solution. Nat. Struct. Mol. Biol., 4, 732-738.

34. Banci,L., Bertini,I., Bren,K.L., Cremonini,M.A., Gray,H.B., Luchinat,C. and Turano,P. (1996) The use of pseudocontact shifts to refine solution structures of paramagnetic metalloproteins: Met80Ala cyano-cytochrome c as an example. JBIC, 1, 117-126.

35. Banci,L., Bertini,I., Huber,J.G., Luchinat,C. and Rosato,A. (1998) Partial orientation of oxidized and reduced cytochrome b5 at high magnetic fields: magnetic susceptibility anisotropy contributions and consequences for protein solution structure determination. J. Am. Chem. Soc., 120, 12903-12909.

36. Cornell,W.D., Cieplak,P., Bayly,C.I., Gould,I.R., Merz,K.M., Ferguson,D.M., Spellmeyer,D.C., Fox,T., Caldwell,J.W. and Kollman,P.A. (1995) A second generation force field for the simulation of proteins, nucleic acids, and organic molecules. J. Am Chem. Soc., 117, 5179-5197.

37. Perez,A., Marchan,I., Svozil,D., Sponer,J., Cheatham,T.E., Laughton,C.A. and Orozco,M. (2007) Refinement of the AMBER force field for nucleic acids: improving the description of $\alpha / \gamma$ conformers. Biophys. J., 92, 3817-3829.

38. Tolbert,B.S., Miyazaki,Y., Barton,S., Kinde,B., Starck,P., Singh,R., Bax,A., Case,D.A. and Summers,M.F. (2010) Major groove width variations in RNA structures determined by NMR and impact of $13 \mathrm{C}$ residual chemical shift anisotropy and $1 \mathrm{H}-13 \mathrm{C}$ residual dipolar coupling on refinement. J. Biomol. NMR, 47, 205-219.

39. Russo Krauss,I., Merlino,A., Randazzo,A., Novellino,E., Mazzarella,L. and Sica,F. (2012) High-resolution structures of two complexes between thrombin and thrombin-binding aptamer shed light on the role of cations in the aptamer inhibitory activity. Nucleic Acids Res., 40, 8119-8128.

40. Lu,X.-J. and Olson,W.K. (2008) 3DNA: a versatile, integrated software system for the analysis, rebuilding and visualization of three-dimensional nucleic-acid structures. Nat. Protoc., 3, 1213-1227.

41. Chen,V.B., Arendall,W.B., Headd,J.J., Keedy,D.A., Immormino,R.M., Kapral,G.J., Murray,L.W., Richardson,J.S. and Richardson,D.C. (2009) MolProbity: all-atom structure validation for macromolecular crystallography. Acta Crystallogr. D Biol. Crystallogr., 66, 12-21.

42. Masiero,S., Trotta,R., Pieraccini,S., De Tito,S., Perone,R., Randazzo,A. and Spada,G.P. (2010) A non-empirical chromophoric interpretation of CD spectra of DNA G-quadruplex structures. Org. Biomol. Chem., 8, 2683-2692.

43. Kim,S.-G., Lin,L.-J. and Reid,B.R. (1992) Determination of nucleic acid backbone conformation by proton NMR. Biochemistry, $\mathbf{3 1}$ 3564-3574.

44. Gorenstein,D.G. (1994) Conformation and dynamics of DNA and protein-DNA complexes by 31P NMR. Chem. Rev., 94, 1315-1338.

45. Markley,J.L., Bax,A., Arata,Y., Hilbers,C.W., Kaptein,R., Sykes,B.D., Wright,P.E. and Wüthrich,K. (1998) Recommendations for the presentation of NMR structures of proteins and nucleic acids (IUPAC Recommendations 1998). Pure Appl. Chem., 70, 117-142.

46. Schultze,P., Macaya,R.F. and Feigon,J. (1994) Three-dimensional solution structure of the thrombin-binding DNA aptamer d(GGTTGGTGTGGTTGG). J. Mol. Biol, 235, 1532-1547.

47. Mao,X., Marky,L.A. and Gmeiner,W.H. (2004) NMR structure of the thrombin-binding DNA aptamer stabilized by Sr2+. J. Biomol. Struct. Dyn., 22, 25-33.

48. Reshetnikov,R., Golovin,A., Spiridonova,V., Kopylov,A. and Šponer,J. (2010) Structural dynamics of thrombin-binding DNA aptamer d(GGTTGGTGTGGTTGG) quadruplex DNA studied by large-scale explicit solvent simulations. J. Chem. Theory Comput., 6 , 3003-3014.

49. Karsisiotis,A.I., Hessari,N.M., Novellino,E., Spada,G.P., Randazzo,A. and Webba da Silva,M. (2011) Topological characterization of nucleic acid G-quadruplexes by UV absorption and circular dichroism. Angew. Chem., 123, 10833-10836.

50. Randazzo,A., Spada,G.P. and da Silva,M.W. (2013) Circular dichroism of quadruplex structures. Top. Curr. Chem., 330, 67-86.

51. Hud,N.V., Smith,F.W., Anet,F.A. and Feigon,J. (1996) The selectivity for $\mathrm{K}+$ versus $\mathrm{Na}+$ in DNA quadruplexes is dominated by relative free energies of hydration: a thermodynamic analysis by $1 \mathrm{H}$ NMR. Biochemistry, 35, 15383-15390.

52. Kankia,B.I. and Marky,L.A. (2001) Folding of the thrombin aptamer into a G-Quadruplex with $\mathrm{Sr} 2+$ : stability, heat, and hydration. J. Am. Chem. Soc., 123, 10799-10804. 